\title{
Assessment of Primary Breakup of Liquid Sheet Emanating from a Pressure Swirl Atomizer for Non-Newtonian Fluids
}

\author{
Krishna Kant ${ }^{\star 1}$, R. Banerjee ${ }^{1}$ \\ ${ }^{1}$ Department of Mechanical and Aerospace, Indian Institute of Technology Hyderabad, \\ Hyderabad, India \\ ${ }^{*}$ Corresponding author email: me17resch11004@iith.ac.in
}

\begin{abstract}
Numerical investigations were performed to determine the breakup length and spray cone angle of liquid sheet emanating from a pressure swirl atomizer. The working fluid was taken to be non-Newtonian in nature whose rheology can be represented as a power law fluid. The sheet morphology during its primary breakup was captured using Coupled Level Set VOF method that was implemented in an in-house two-phase Navier Stokes solver based on OpenFOAM libraries. The flow was assumed to be 2D axisymmetric.

A parametric study was performed to determine the effect of injector geometry parameters like contraction ratio $R_{S} / R_{o}$, orifice length $L_{o} / R_{o}$ and contraction angle $\beta$; injector operating condition like pressure drop across the injector $\Delta p$, density ratio $\rho_{l} / \rho_{g}$ and inlet swirl ratio $S R=$ $u_{x} / u_{\theta}$. Both shear thinning fluid and shear thickening fluids was investigated by varying the power law index, $n$, between 0.5 to 1.5 . The sheet breakup and its dynamics for nonNewtonian fluid will be compared with Newtonian fluid breakup mechanism.
\end{abstract}

\section{Keywords}

Pressure swirl atomizer, Non-Newtonian fluid, Primary Atomization, CLSVOF, OpenFOAM.

\section{Introduction}

Spray and atomization of sheet-based atomization sees application in several engineering applications. The flow physics is governed by number of forces namely aerodynamic force, viscous force, surface tension force, liquid centrifugal force, inertial force, turbulence and sometimes cavitation. The main key parameters that plays the role in internal flow characteristic of atomizers are atomizer constant, contraction ratio $R_{S} / R_{O}$ and length of the orifice $L_{o}[1]$. Based on these parameters and pressure drop $\Delta p$, several correlations had been proposed for the spray cone angle, breakup length, droplet size distribution and liquid film thickness. However, these correlations are majorly obtained from experimental data for Newtonian fluids. According to Ashgriz [2], the breakup of the conical sheet from pressure swirl atomizer (PSA) happens due to sheet perforation, flapping of sheet by fluctuation of the air core vortex and growth of instabilities wave generated by aerodynamic forces. In a recent work by Ibrahim and Jog, [3,4] a detailed numerical and analytical study was performed for annular liquid sheet atomization. They analysed the effect of liquid viscosity and operating pressure for Newtonian fluid through numerical simulation. From the same research group Mandal et. al. [5] extended the work for power law fluids and had reported that film thickness and discharge coefficient increases while spray cone angle decreases with power law index $n$. Numerous other works can be found on PSA but combined study of internal flow and spray characteristic is rare [6].

In the present investigation, a combined study of internal flow of nozzle and primary atomization has been performed. For instance, the spray cone angle reported in previous work (Mohammad, et. al. [7], Ibrahim and Jog [4]) estimated the spray angle at the nozzle exit using 
the formula $\theta=2 \tan ^{-1}\left(u_{r} / u_{x}\right)$. However, this expression overestimates the spray cone angle than what is determined experimentally. This may be because the liquid spray sheet interacts with the ambient gas and vortices are formed after emanating from the nozzle leading to a reduced spray cone angle. In the present work, spray cone angle and breakup length are directly estimated from the CFD solutions because detailed interface tracking is performed in the primary breakup regime using CLSVOF method. It was found that the spray cone angle estimated from the current work differs by $10-20^{\circ}$ from the aforementioned method. The present work also discusses the role of different parameters in primary breakup, vortex interaction with liquid and gas, sheet flapping and droplet collisions with the mother sheet, which were not investigated in previous works. This work is more in line with the work of Laurila et. al. [6] but with larger sets of parameters and also considers rheology of non-Newtonian fluid. The sheet morphology during its primary breakup was captured using Coupled Level Set VOF method that was implemented in an in-house two-phase Navier Stokes solver based on OpenFOAM libraries. The flow is assumed to be $2 \mathrm{D}$ axisymmetric. A parametric study was performed to determine the effect of injector geometry parameters like contraction ratio $R_{S} / R_{o}$, orifice length $L_{o} / R_{o}$ and contraction angle $\beta$; injector operating condition like pressure drop across the injector $\Delta p$, density ratio $\rho_{l} / \rho_{g}$ and swirl ratio $S R=u_{x} / u_{\theta}$. Both shear thinning fluid and shear thickening fluids was investigated by varying the power law index, $n$, between 0.5 to 1.5. The sheet breakup and its dynamics for non-Newtonian fluid will be compared with Newtonian fluid breakup mechanism.

\section{Solver Description}

\section{Coupled Level Set and VOF Formulation}

The isoAdvector scheme proposed by [8] was used in this work. The governing equation for volume fraction $\alpha$ is given as

$$
\frac{\partial \alpha}{\partial t}+\nabla \cdot(\vec{u} \alpha)=0
$$

where, $\alpha$ is 1 for liquid, 0 for gas and in between 0 and 1 at interface. The level set scalar, $\phi$ is a distance function which is explicitly initialized from the VOF function using $\phi_{0}=(2 \alpha-$ 1) $\times 0.75 \Delta_{m}$ [9]. $\Delta_{m}$ is the minimum value from $\Delta x, \Delta y$ and $\Delta z$. For smooth curvature field, it is required that $|\nabla \phi|=1$ near interface. To maintain that distance property a re-distancing equation is solved using a pseudo time step $\tau=0.1 \Delta_{m}$.

$$
\frac{\partial \phi}{\partial \tau}+S \times(\nabla \phi-1)=0
$$

where, $S$ is the sign function defined as $S=\phi_{0} /\left(\phi_{0}^{2}+|\nabla \phi|^{2} \Delta_{m}^{2}\right)$.

\section{Curvature Calculation}

For the reduction of spurious currents it is required that the volume fraction $\alpha$ and the interface normal vector $\vec{n}=\nabla \phi /|\nabla \phi|$ are as smooth as possible since surface tension force is modelled as $f_{S}=\sigma \nabla \alpha C$, where $C=-\nabla \cdot \vec{n}$ while using the CSF model [10]. Initially, interface normal vector is smoothed by interpolating it to cell vertices and then back to cell centre through inverse distance function [11]. In a uniform 2D mesh the above said interpolation routine results in

$$
\vec{n}_{P}^{\prime}=\frac{1}{4} \vec{n}_{P}+\frac{1}{8}\left(\vec{n}_{N}+\vec{n}_{S}+\vec{n}_{E}+\vec{n}_{W}\right)+\frac{1}{16}\left(\vec{n}_{N W}+\vec{n}_{N E}+\vec{n}_{S W}+\vec{n}_{S E}\right)
$$

Thereafter, the iso-surface of $\phi=0$ is determined for each cell near the vicinity of the liquid/gas interface. The curvature value for a cell is equated to the curvature value of its 
corresponding closest point to the interface [12]. Thus reducing the variation of curvature with distance from interface. Curvature value on the iso-surface points are calculated through interpolation of the curvature field $C^{\prime}=-\nabla \cdot n^{\prime}$ using the mixed linear weighted cell-face interpolation.

$$
C^{*}=\frac{w_{1} C_{i}^{\prime}+\sum w_{2} C_{i F}^{\prime}}{\left(w_{1}+\sum w_{2}\right)}
$$

where, $C_{i}^{\prime}$ and $C_{i F}^{\prime}$ are the curvature at the cell centre and cell faces of the cell at which the iso-surface point lie. The weights $w_{1}$ and $w_{2}$ are calculated based on the distance between so-surface point and the cell centre and cell faces respectively. The smoothed surface tension force is modelled as $f_{s}=\sigma \nabla \alpha C^{*}$.

Non-Newtonian Fluid Modelling

Power law based non-Newtonian viscosity model was used in present work. The kinematic viscosity of liquid is estimated by

$$
v_{l}=K|\dot{\gamma}|^{n-1}
$$

where, $K$ and $n$ are the model constants and strain rate $|\dot{\gamma}|=2 \sqrt{D: D} \cdot D=\frac{1}{2}\left(\nabla \vec{U}+\nabla \vec{U}^{T}\right)$ is the strain rate tensor. To avoid the problem of singularity, viscosity is bounded by maximum and minimum value.

\section{Transport Equations}

For incompressible two phase flows, the governing equation of conservation of mass and momentum are as follows:

$$
\begin{aligned}
& \nabla \cdot \vec{u}=0 \\
& \begin{aligned}
\frac{\partial \rho \vec{u}}{\partial t}+\nabla \cdot(\rho \vec{u} \vec{u}) & -\nabla \cdot\left[\left(\mu+\mu_{S G S}\right)\left(\nabla \vec{u}+\nabla \vec{u}^{T}-\frac{2}{3}(\nabla \cdot \vec{u}) I\right)\right] \\
& =-\nabla p_{d}^{*}-g(\vec{x} \cdot \nabla \rho)+\vec{F}_{\sigma}
\end{aligned}
\end{aligned}
$$

Where, $\vec{u}$ is the Filtered velocity defined as $\vec{u}=\alpha \vec{u}_{l}+(1-\alpha) \vec{u}_{g}, \vec{u}_{l}$ and $\vec{u}_{g}$ being the filtered liquid and gas velocity. $\mu_{S G S}$ is the sub-grid scale viscosity and $p_{d}^{*}$ is the modified dynamic pressure defined as $p_{d}^{*}=p-\rho(g \cdot \vec{x})+\frac{1}{3} \operatorname{tr}\left(\tau_{t}\right) . \vec{x}$ is the distance vector from the reference pressure point and $\tau_{t}$ is the modelled turbulent stress. Fluid properties are calculated as the weighted average of volume fraction $\alpha$ i.e. $\rho=\rho_{l} \alpha+\rho_{g}(1-\alpha)$ and $\mu=\mu_{l} \alpha+\mu_{g}(1-\alpha)$. In the present work turbulent stresses and turbulent viscosity are modelled through dynamic kequation sub-grid LES model [13]. It is a one equation model where sub-grid scale kinetic energy $k_{S G S}$ is solved through the equation

$$
\frac{\partial k_{S G S}}{\partial t}+\nabla \cdot\left(\vec{u} k_{S G S}\right)=-\tau \cdot \nabla \vec{u}-\varepsilon+\nabla\left(v_{T} \nabla k_{S G S}\right)
$$

Here, $\tau$ is the turbulent stress calculated as $\tau=-v_{T}\left(\nabla \vec{u}+\nabla \vec{u}^{T}\right)+\frac{2}{3} \delta k_{S G S}$ where $\delta$ is a Dirac function. $\varepsilon$ is the dissipation term given by $\varepsilon=C_{\varepsilon} \frac{k_{S G S}^{3 / 2}}{\Delta}$ and turbulent viscosity $v_{T}=C_{v} k_{S G S}^{1 / 2} \Delta$ where $C_{\varepsilon}$ and $C_{v}$ are the model constants. Characteristic length $\Delta$ is determine by cube root of volume as for its consistency. A simple averaging of Gaussian function is used as the filter kernel in the present work. The above governing equations are solved using the finite volume method in OpenFOAM [14,15]. A second order accurate spatial and time discretization 
schemes are used in the present work. The details on the discretization and matrix solvers are summarized in Table 1. Further details on the in-house solver can be found in [16].

Table 1 - Discretization schemes and matrix solution algorithm used in the current work

\begin{tabular}{|c|c|c|c|c|c|}
\hline Equations & $\begin{array}{l}\text { Divergence } \\
\text { Scheme }\end{array}$ & $\begin{array}{l}\text { Laplacian } \\
\text { Scheme }\end{array}$ & Linear Solver & Pre-Conditioner & Tolerence \\
\hline Eqn. 1. & VanLeer & NA & smoothSolver & symGaussSeidel & $10^{-8}$ \\
\hline Eqn. 2. & NA & NA & Explicit & NA & 0 \\
\hline Eqn. 7. & limitedLinearV & $\begin{array}{l}\text { Gauss } \\
\text { Linear }\end{array}$ & SmoothSolver & symGaussSeidel & $10^{-6}$ \\
\hline Eqn. 6. & NA & $\begin{array}{l}\text { Gauss } \\
\text { Linear }\end{array}$ & GAMG & DIC & $10^{-8}$ \\
\hline Eqn. 8. & limitedLinear & $\begin{array}{l}\text { Gauss } \\
\text { Linear }\end{array}$ & smoothSolver & symGaussSeidel & $10^{-8}$ \\
\hline
\end{tabular}

\section{Problem Setup and Formulation}

Simulation performed were performed on a 2D axisymmetric geometry as shown in Figure 1. For the present study, the power law index is varied from $0.5<n<1.5$. The properties of the fluids are tabulated in Table 2. The surrounding fluid always remains pressurized air and their properties are shown in Table 3. The current work focuses on the effect of the nozzle geometry and the operating condition on the primary breakup of the spray. The base case test matrix is shown in Table 4 which is defined by 8 independent parameters of which 7 are altered and discussed in the later sections. The chosen parameters are based on a commercially available PSA nozzle and used in a previous experimental study [17]. The mesh is generated with nonuniform cells with an expansion ratio of 5 and $R_{o} / \Delta=60$ such that for the first cell width at $\operatorname{Re} \sim 10^{7} y^{+} \sim 0.5$.

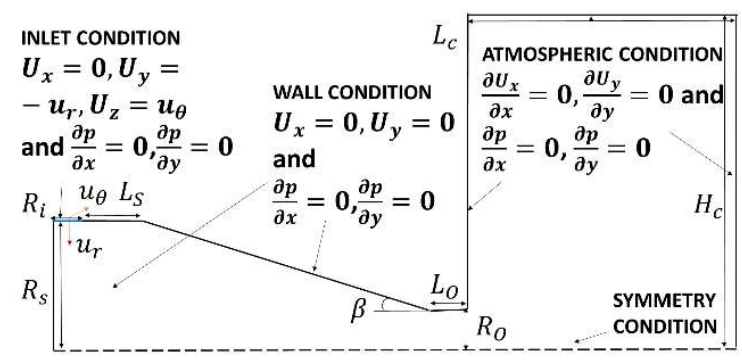

Figure 1. Schematic of 2D axisymmetric pressure swirl atomizer

Table 2 - Properties of liquids

\begin{tabular}{|c|c|c|c|c|c|c|}
\hline \multirow{2}{*}{$\begin{array}{l}\text { Fluid } \\
\text { Name }\end{array}$} & \multicolumn{4}{|c|}{ Power Law Model Constants } & \multicolumn{2}{|c|}{ Material Properties } \\
\hline & $\mathrm{K}($ Pa.s $)$ & $n$ & $\begin{array}{c}\mu_{\max } \\
(\text { Pa.s })\end{array}$ & $\begin{array}{c}\mu_{\min } \\
(\text { Pa.s })\end{array}$ & $\begin{array}{l}\text { Density } \rho_{l} \\
\left(\mathrm{Kg} / \mathrm{m}^{3}\right)\end{array}$ & $\begin{array}{c}\text { Surface } \\
\text { Tension } \\
\text { Coefficient } \\
\sigma(m N / m) \\
\end{array}$ \\
\hline Newtonian & $10^{-3}$ & 1.0 & $10^{-3}$ & $10^{-3}$ & 1000 & 72 \\
\hline $\begin{array}{l}\text { Shear } \\
\text { Thinning }\end{array}$ & $10^{-2}$ & 0.5 & $10^{-3}$ & $10^{-4}$ & 1000 & 72 \\
\hline $\begin{array}{l}\text { Shear } \\
\text { Thickening }\end{array}$ & $10^{-4}$ & 1.5 & $10^{-2}$ & $10^{-3}$ & 1000 & 72 \\
\hline
\end{tabular}

Table 3 - Properties of surrounding fluid

\begin{tabular}{c|ccc}
\hline Property & Case 1 & Case 2 & Case 3 \\
\hline Density $\rho_{g}\left(\mathrm{Kg} / \mathrm{m}^{3}\right)$ & 1.272 & 12.72 & 100 \\
Viscosity $\mu_{g}(\mathrm{~Pa} \cdot \mathrm{s})$ & $1.85 \times 10^{-5}$ & $1.85 \times 10^{-5}$ & $1.85 \times 10^{-5}$
\end{tabular}


Table 4 - Parameters for the base test case where fluid used is Newtonian and gas is Air 3 with the nozzle radius $R_{O}=1 \mathrm{~mm}$

\begin{tabular}{ccccccccc}
\hline & \multicolumn{3}{c}{ Geometrical parameters } & \multicolumn{3}{c}{ Flow Parameters } \\
$\frac{R_{i}}{R_{o}}$ & $\frac{A_{i}}{D_{S} D_{o}}$ & $\frac{R_{s}}{R_{o}}$ & $\beta\left(\right.$ in $\left.^{\circ}\right)$ & $\frac{L_{s}}{R_{o}}$ & $\frac{L_{o}}{R_{o}}$ & $\mathrm{Kg} / \mathrm{hr})$ & $\frac{u_{\theta}}{u_{r}}$ & $\frac{\rho_{l}}{\rho_{g}}$ \\
\hline 0.18 & 1.1 & 1.8 & 38.5 & 0.018 & 1.5 & 0.49 & 1.11 & $10^{1}$ \\
& $\times 10^{-3}$ & & & & & & &
\end{tabular}

\section{Results and Discussion}

Depending on the pressure drop across a PSA, five types of spray structures can be observed dribble, distorted pencil, onion, tulip and cone [1]. Due to the tangential inlet velocity, which is parameterized by the inlet swirl number as $u_{\theta} / u_{r}$, the liquid is constrained within the peripheral region of the nozzle. Both the axial and tangential velocities of the liquid increases when it negotiates the conical region of the nozzle due to acceleration of the fluid due to smaller crosssection area and conservation of angular momentum, respectively. The jet finally emanates from the orifice exit forming a tulip structure. Figure 2 shows the essential flow features of the spray in the primary breakup region at two different non-dimensional times $\left(\tau=u_{r, i} t / D_{o}\right)$. Vortices are formed inside the liquid at the contraction region which accounts for losses of kinetic energy. In the gaseous phase, vortices are observed in the air core region inside the nozzle. These vortices start growing in the axial direction till the point of liquid breakup. Thus, a strong recirculation zone exists near the sheet breakup region as shown in the Figure 2. Apart from these vortices, a number of small vortices also exist near the droplets and ligaments. As seen in Figure 2, these vortices grow with the passage of time as well. The KH instability waves get generated in the jets from the shear forces acting at interface between liquid and air-core region. The Weber number $\left(W e_{l}=\frac{\rho_{l} U_{o}^{2} D_{0}}{\sigma}=2546\right)$ and the Reynolds number $\left(R e_{l}=\frac{\rho_{l} U_{o} D_{0}}{\mu_{l}}=19.15 \times 10^{6}\right)$ of the liquid sheet emanating is quite high. The flapping of the liquid sheet is observed under these conditions and is quantified through Strouhal number $S t=f D_{o} / U_{o}$. The time history of cone angle and its conversion into the frequency domain by performing an FFT on the time series data is shown in Figure 3. The Strouhal number for maximum frequency $f=120 \mathrm{~Hz}$ is found to be 25.8 . Finally, the jets break by the growth of the instability waves and flapping of the sheet. The ligaments formed by flapping action are of larger size than the blobs formed by breaking from instability waves. Thus wide variation of drop sizes are generated. The breakup length varies significantly because the liquid blobs generated tends to drift back and coalesce with the liquid sheet due to the large recirculation vortices in the vicinity of the primary breakup region.

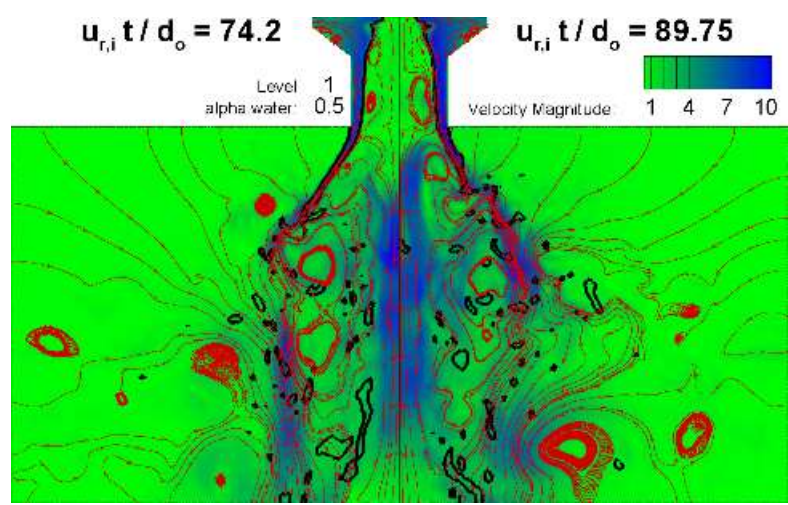


Figure 2. Streamlines shown as red lines are for the case given in Table 4 using radial $u_{r}$ and axial $u_{x}$ velocities. The colour contour represents the velocity magnitude $U=\sqrt{u_{x}^{2}+u_{r}^{2}+u_{\theta}^{2}}$. Left half is at $\frac{u_{r, i} t}{D_{o}}=74.2$ and right half for $\frac{u_{r, i} t}{D_{o}}=89.76$ time step.
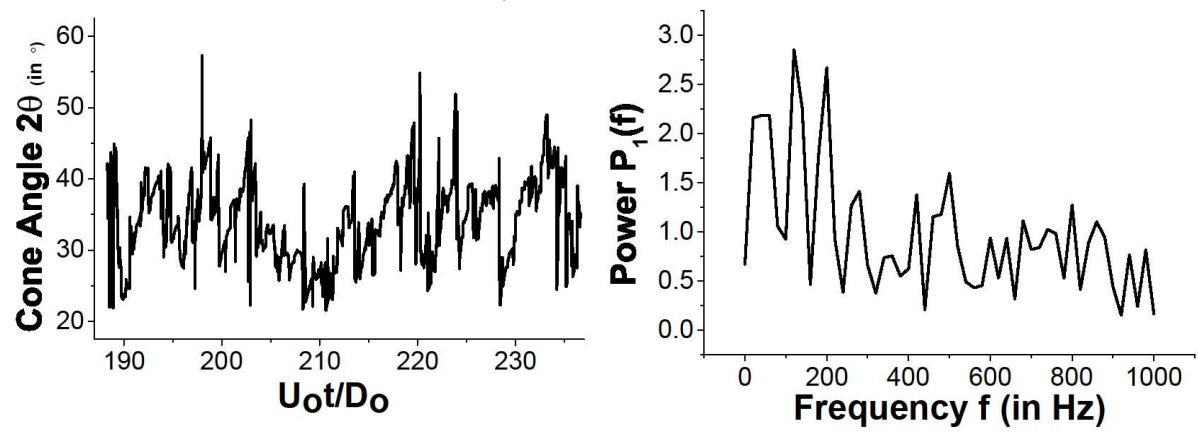

Figure 3. Cone angle variation with respect to non-dimensional time $U_{o} t / D_{o}$ and its FFT analysis in temporal domain.

\section{Effect of Operating parameters}

The major operational parameters that effect the spray characteristic are inlet swirl number $u_{\theta} / u_{r}$, density ratio $\rho_{l} / \rho_{g}$ and mass flow rate $\dot{Q}$. The internal flow and spray characteristic parameters variation along with various operating parameters are shown in Figure 4. Increase in inlet swirl ratio raises the centrifugal force of the liquid and thus raising the spray cone angle and reducing the film thickness. Since film thickness and orifice velocity are inversely related by conservation of mass so reduction in film thickness cause increase in orifice velocity as shown in Figure 4. Also the breakup length and cone angle are inversely related thus breakup length decreases with increase in inlet swirl ratio. The increase in density of air increases the drag force on liquid sheet and cause reduction in breakup length and increase of spray cone angle which is shown in Figure 4. However a critical value is observed for the density ratio close to $O\left(10^{2}\right)$ beyond which the effect is negligible. Density ratio has negligible effect on internal flow characteristics. Sheet perforation phenomenon is observed only in high density ratio cases. Mass flow rate and pressure drop across the atomizer are related to each other. For the given parameters the rise in mass flow rate changed the pressure from 0.5 bar to 1.8 bar. However, the flow structure remains the same for all cases and thus similar internal and spray characteristics are observed in Figure 4. The effect of power law index for nonNewtonian fluid is governed by strain rate. In primary atomization strain rate is very high i.e. in the range of the order of $\left(10^{4}-10^{6}\right)$, proportional to liquid orifice velocity $U_{o}$ and inversely proportional to film thickness $h$. Thus the spray characteristic for non-Newtonian fluids is found close to the Newtonian fluid in the limit of higher strain rate end. The reduction in dissipation energy by reducing viscosity of liquid for shear thinning fluid also increases the spray cone angle and breakup length and vice versa for shear thickening fluid. Breakup length and spray cone angle are calculated from the post processing tools which utilizes the similar techniques as described in [17]. Orifice data are extracted for more than 100 time steps to estimate film thickness and orifice velocity.
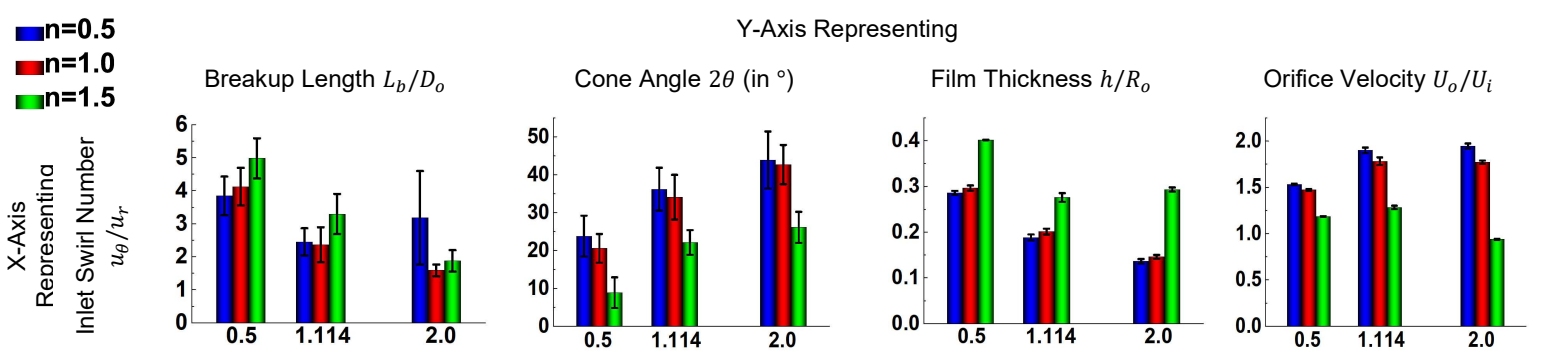

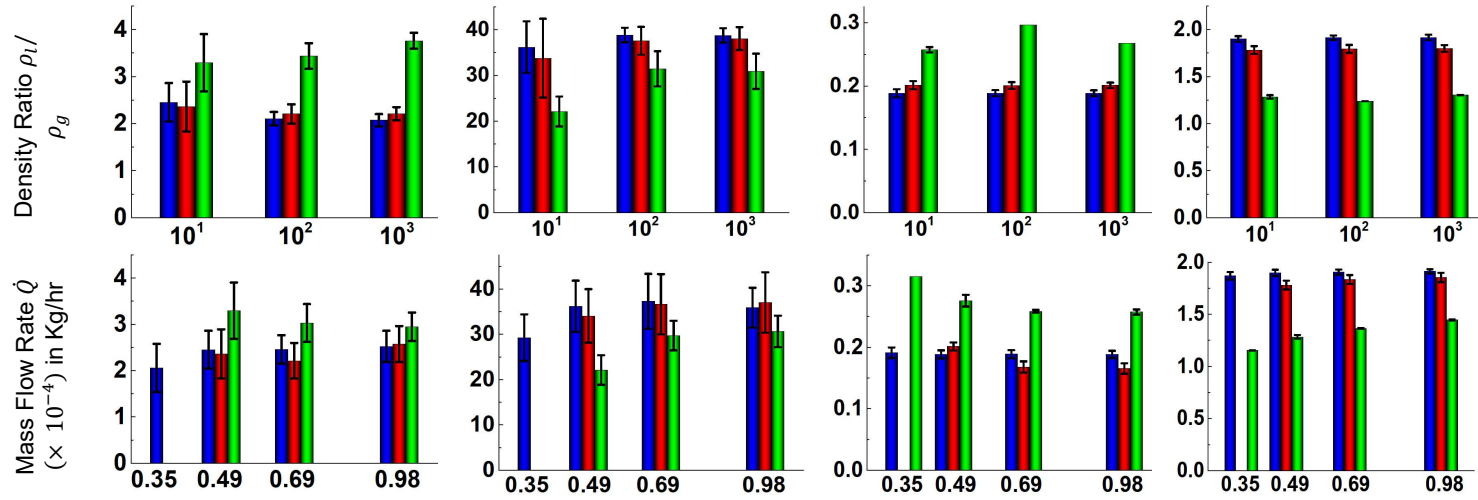

Figure 4. Effect of different operating parameters such as inlet swirl number, density ratio and mass flow rate $\dot{Q}$ on the internal and external spray characteristics. The standard deviation is calculated from over 100 frames.

\section{Effect of Nozzle geometry}

The spray characteristic of the swirl nozzle is effected by many geometrical parameters such as atomizer constant $\frac{A_{i}}{D_{S} D_{o}}$, contraction ratio $R_{S} / R_{o}$, contraction angle $\beta$ and length of orifice $L_{o} / R_{o}$. The contraction ratio has the most significant influence on the spray morphology because as the contraction ratio increases, the kinetic energy of the fluid correspondingly increases. Thus the spray cone angle and breakup length increases with the increase in contraction ratio and is shown in Figure 5. The ratio of momentum at nozzle exit to nozzle entrance is proportional to contraction ratio. However due to high viscous losses in shear thickening fluid, this ratio reduces significantly. It is also observed that for very high contraction ratio, the losses from vortex formation in liquid also raises. Thus $U_{o} / U_{i}$ is much less than $R_{S} / R_{o}$. Next, as the contraction angle increases pressure gradient increases thus increasing the acceleration of fluid. The length of the contraction region decreases with the increase in contraction thus reducing the pressure losses. However, with the sudden contraction the losses due to formation of vortex increases. Due to this, conflicting nature there exist a maxima of energy loss near to $\beta=45^{\circ}$ and the spray cone angle and breakup are observed to be minimum for this configuration. Figure $\mathbf{5}$ shows the various internal flow and spray characteristic with varying contraction angle. The increase in the orifice length increases the pressure loss thus causing a reduction in the kinetic energy and a decrement in spray cone angle and breakup length is observed as shown in Figure $\mathbf{5}$.

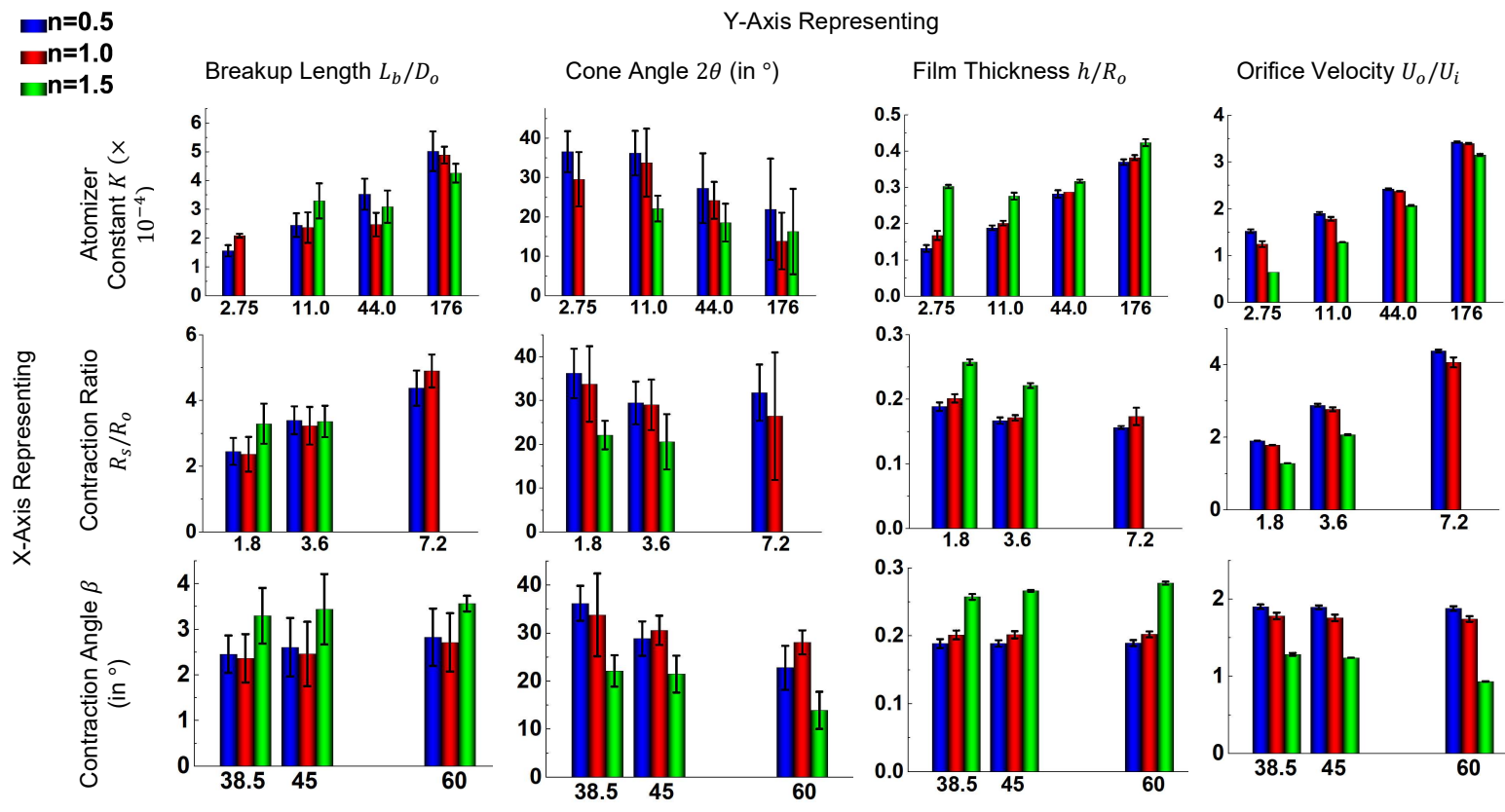



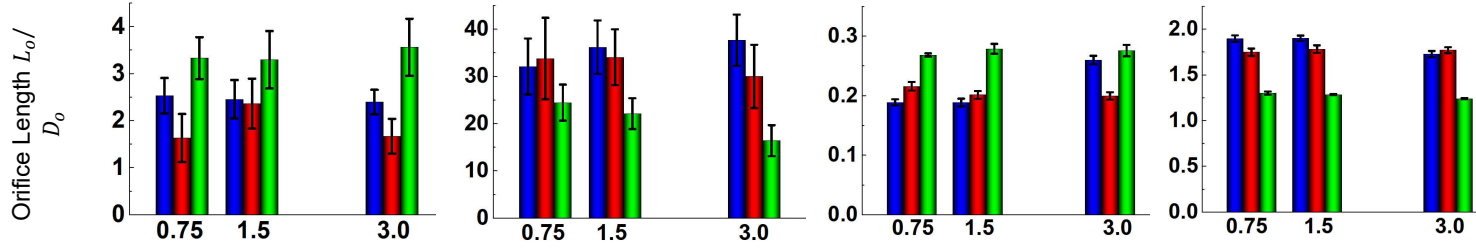

Figure 5. Effect of internal geometry of nozzle on spray's internal and external characteristics. The standard deviation is calculated from over 100 frames.

\section{Conclusions}

For the cases performed in these work the pressure difference is $0.5-1.8$ bar. Weber number and Reynolds number for the liquid jet emanating are of the order $10^{3}$ and $10^{7}$ respectively. Under these condition the strain rate observed is quite high and the liquid behave closer to the higher strain rate end i.e. lower viscosity for shear thinning and high viscosity for shear thickening. At low density ratio onion and tulip structures are majorly observed while fully developed cone is observed for high density ratio $\left(>10^{2}\right)$. The standard deviation for cone angle is $20-30 \%$ while for breakup length is $10-20 \%$. On the other hand, standard deviation for film thickness and orifice velocities are observed to be around $1-3 \%$.

\section{Acknowledgments}

The authors would like to acknowledge the financial support by Ministry of Education, Government of India to Mr K Kant, Mr. Madhu for his technical support in managing the computational hardware and software and Dr. Harish N. Dixit for providing access to computational resources from his group to simulate the cases reported in this work.

\section{Nomenclature}

PSA Pressure Swirl Atomizer

$U_{o}, U_{i}$ Velocity magnitude $\sqrt{u_{x}^{2}+u_{r}^{2}+u_{\theta}^{2}}$ at nozzle exit and inlet respectively

\section{References}

[1] Lefebvre, A. H., and McDonell, V. G., 2017, Atomization and Sprays, CRC Press.

[2] Ashgriz, N., 2011, Handbook of Atomization and Sprays: Theory and Applications, Springer US.

[3] Ibrahim, A. A., and Jog, M. A., 2008, "Nonlinear Instability of an Annular Liquid Sheet Exposed to Gas Flow," Int. J. Multiph. Flow, 34(7), pp. 647-664.

[4] Ibrahim, A., and Jog, M., 2006, "3 D Simulation of Flow in Pressure-Swirl Atomizers."

[5] Mandal, A., Jog, M. A., Xue, J., and Ibrahim, A. A., 2008, "Flow of Power-Law Fluids in Simplex Atomizers," Int. J. Heat Fluid Flow, 29(5), pp. 1494-1503.

[6] Laurila, E., Roenby, J., Maakala, V., Peltonen, P., Kahila, H., and Vuorinen, V., 2019, "Analysis of Viscous Fluid Flow in a Pressure-Swirl Atomizer Using Large-Eddy Simulation," Int. J. Multiph. Flow, 113, pp. 371-388.

[7] Rezaeimoghaddam, M., Elahi, R., Modarres Razavi, M. R., and Ayani, M. B., 2010, "Modeling of Non-Newtonian Fluid Flow Within Simplex Atomizers," pp. 549-556.

[8] Roenby, J., Bredmose, H., and Jasak, H., 2021, "A Computational Method for Sharp Interface Advection," R. Soc. Open Sci., 3(11), p. 160405.

[9] Albadawi, A., Donoghue, D. B., Robinson, A. J., Murray, D. B., and Delauré, Y. M. C., 2013, "Influence of Surface Tension Implementation in Volume of Fluid and Coupled Volume of Fluid with Level Set Methods for Bubble Growth and Detachment," Int. J. Multiph. Flow, 53, pp. 1128.

[10] Brackbill, J. U., Kothe, D. B., and Zemach, C., 1992, "A Continuum Method for Modeling Surface Tension," J. Comput. Phys., 100(2), pp. 335-354.

[11] Haghshenas, M., Wilson, J. A., and Kumar, R., 2017, "Algebraic Coupled Level Set-Volume of 
Fluid Method for Surface Tension Dominant Two-Phase Flows," Int. J. Multiph. Flow, 90, pp. 13-28.

[12] Coquerelle, M., and Glockner, S., 2016, "A Fourth-Order Accurate Curvature Computation in a Level Set Framework for Two-Phase Flows Subjected to Surface Tension Forces," J. Comput. Phys., 305, pp. 838-876.

[13] Kim, W.-W., and Menon, S., "A New Dynamic One-Equation Subgrid-Scale Model for Large Eddy Simulations," 33rd Aerospace Sciences Meeting and Exhibit.

[14] Jasak, H., 2009, "OpenFOAM: Open Source CFD in Research and Industry," Int. J. Nav. Archit. Ocean Eng., 1(2), pp. 89-94.

[15] Deshpande, S. S., Anumolu, L., and Trujillo, M. F., 2012, "Evaluating the Performance of the Two-Phase Flow Solver InterFoam," Comput. Sci. Discov., 5(1), p. 14016.

[16] Kant, K., Kumar, M., Reddy, R., Banerjee, R., Mangadoddy, N., and Vanka, S., 2019, "Numerical Study of Primary Jet Breakup in a Simplex Swirl Atomizer Using Dual Grid Coupled Level Set VOF Method."

[17] Michael, E., Keerthi, S. K., Kant, K., Kolhe, P., Banerjee, R., and Chakravarthy, S. R., 2019, "Effect of Liquid/Gas Density Ratio on Primary Jet Breakup of Pressure Swirl Atomizer: Experimental and Numerical Study." 